\title{
CONSUMO ELEVADO DE ANTIDEPRESSIVOS E SUAS MOTIVAÇÕES
}

\section{HIGH CONSUMPTION OF ANTIDEPRESSANTS AND THEIR MOTIVATIONS}

\author{
Cleidiane Mares Costa Cabral \\ Bacharel em Farmácia pela Faculadade de Almeanara - ALFA. \\ E-mail:cleidy.mares@gmail.com
}

Mônica Cecília Santana Pereira

Graduação em Farmácia-Bioquímica pela Universidade Federal de Juiz de Fora; Especialista em Vigilância Sanitária e legislação pela Universidade Federal do Rio de Janeiro; Mestre em Ciência e Tecnologia do Leite e derivados pelo Mestrado Profissional em Ciência e Tecnologia do Leite e derivados da Universidade Federal de Juiz de Fora.

. Doutora em Promoção de Saúde pela Universidade de Franca/Cruzeiro do Sul. E-mail: monicasantanapereira@bol.com.br

\section{Viviane Amaral Toledo Coelho}

Bióloga pelo Centro de Ensino Superior de Juiz de Fora; Especialista em Solos e Meio Ambiente pela Universidade Federal de Lavras; Mestre e Doutora em Ciência do Solo pela Universidade Federal de Lavras. Docente da Faculdade de Almenara - ALFA de Almenara - Minas Gerais. E-mail: vivianeatc@yahoo.com.br

\section{Luiza Gobira Lacerda}

Graduação em Farmácia e Habilitação em Bioquímica pela Fundação Universidade de Itaúna; Especialização em Farmacologia e Interação Medicamentosas pelo

Centro Universitário Internacional; Docente da Faculdade de Almenara - ALFA de Almenara - Minas Gerais.

E-mail: lugobila@hotmail.com 
Graduado em Pedagogia pela Universidade Estadual de Montes Claros; Pósgraduação em Psicopedagogia Clínica e Institucional com Ênfase em Educação Especial e Inclusiva pela Faculdade Einstein; Mestre pelo Programa de Mestrado Profissional em Educação Tecnológica do IFTM - Campus Uberaba-MG Pedagogo no IFNMG - Campus Almenara. E-mail: ronivaldoferreiramendes@gmail.com

\section{RESUMO}

A depressão assume um índice de alta prevalência no mundo, estimam-se que a prevalência deste transtorno na população mundial era de cerca de $14 \%$, e que no Brasil subia para $18,4 \%$, a maior porcentagem entre os países em desenvolvimento e a terceira em termos globais. $O$ objetivo trabalho é identificar as razões que motivam à adesão dos pacientes aos medicamentos indicados para tratamento de transtornos depressivos. A metodologia utilizada foi revisão de literatura onde foram selecionados trinta e quatro artigos. Os estudos foram definidos artigos publicados em português entre janeiro de 2010 a dezembro de 2018, com abordagem envolvendo a depressão (conceito, sintomas e tratamento) e o uso de antidepressivos. Conclui-se que as razões que motivam o uso elevado de antidepressivos tais como: aumento do envelhecimento da sociedade e as condições sociodemográficas da população; elevados números de prescrições de antidepressivos desnecessários; ampliação das indicações dos antidepressivos para outros agravos que não sejam o transtorno depressivo e a medicalização onde a sociedade procura anular o sofrimento com a terapia medicamentosa.

Palavras-chave: Depressão. Antidepressivos. Assistência farmacêutica.

\section{ABSTRACT}

Depression assumes a high prevalence rate in the world, it is estimated that the prevalence of this disorder in the world population was around 14\%, and that in Brazil it rose to $18.4 \%$, the highest percentage among developing countries and the third globally. The objective of this work was this study aims to identify the reasons that 
motivate patients' adherence to the drugs indicated for the treatment of depressive disorders. The methodology used was a literature review where thirty-four articles were selected. As inclusion criteria for screening studies, articles published in Portuguese between January 2010 and December 2018 were defined, with an approach involving depression (concept, symptoms and treatment) and the use of antidepressants. Repeated articles, abstracts, dissertations and theses were excluded. It is concluded that the reasons that motivate the high use of antidepressants such as: increase in the aging of society and the sociodemographic conditions of the population; high numbers of unnecessary antidepressant prescriptions; expansion of indications for antidepressants for other conditions other than depressive disorder and medicalization, where society seeks to eliminate suffering with drug therapy.

Key words: depression, antidepressants and pharmaceutical assistance.

\section{INTRODUÇÃO}

A depressão não é apenas um sentimento de tristeza, mas trata se de um estado patológico que necessita de tratamento farmacológico, o transtorno depressivo possui diagnostico complexo. Segundo a associação americana de psiquiatria de forma geral, os transtornos depressivos são caracterizados por manifestações afetivas consideradas inadequadas em termos de intensidade, frequência e duração do quadro comportamental (ASSOCIAÇÃO AMERICANA DE PSIQUIATRIA, 2014).

Este estado de debilidade reconhecido como depressão assume um índice de alta prevalência no mundo, estimam-se que a prevalência deste transtorno na população mundial era de cerca de $14 \%$, e que no Brasil subia para $18,4 \%$, a maior porcentagem entre os países em desenvolvimento e a terceira em termos globais (ANTUNES, MONICO, 2015).

O transtorno depressivo tem maior prevalência em mulheres, e apesar do diagnóstico complexo, existem critérios que direcionam e ajudam a diagnosticar a patologia neurológica, tendo como parâmetro para o diagnóstico a constatação de humor deprimido e anedonia (perda da capacidade de sentir prazer na execução de atividades antes prazerosas) por duas semanas, em associação com mais de quatro 
sintomas, como: sentimento de desesperança, desvalia, culpa, desamparo, alterações no apetite e sono, fadiga retarda ou agitação psicomotora, diminuição do desempenho sexual, dificuldade de concentração e raciocínio, pensamentos recorrentes sobre morte, com ou sem tentativa de suicídio (FERREIRA et al., 2017).

A terapia farmacológica usada nos transtornos depressivos é uma forma eficaz do tratamento para depressão moderada-grave, mas não é a primeira linha de tratamento para casos de depressão leve. Existem três processos clínicos pelos quais o paciente depressivo passa: a detecção, a medicação psicotrópica e o aconselhamento, o tipo de tratamento depende da gravidade da doença, sendo que a simples detecção não garante uma intervenção adequada. Relativamente à medicação, refiram-se os dois tipos de psicofármacos mais comumente usados em pacientes com depressão: os antidepressivos e os tranquilizantes (PADILHA et al., 2014).

O terceiro processo clínico seria então o aconselhamento, a Psicoterapia. $O$ tratamento com fármacos psicotrópicos é definido como aqueles que afetam o humor e o comportamento, onde agem alterando a comunicação entre os neurônios produzindo diversos efeitos de acordo com o tipo de neurotransmissor comprometido e a forma como a droga atua. Dessa forma, de acordo com o tipo de ação, as drogas podem provocar euforia, ansiedade, sonolência, alucinações, delírios etc. A ação de cada psicotrópico depende: da classe do fármaco, da via de administração, da quantidade, do tempo, da frequência de uso, da absorção e eliminação desta pelo organismo e também da associação com outros fármacos (MAGALHÃES et al., 2016).

O uso abusivo de determinados medicamentos no Brasil cresceu de forma exorbitante, como medida de controle e segurança foi editada e publicada a RDC nํㅜ 27 de 30 de março de 2007, que instituiu o Sistema Nacional para Gerenciamento de Produtos Controlados (SNGPC). O SNGPC tem como principais objetivos: monitorar a dispensação de medicamentos e substâncias entorpecentes e psicotrópicas e seus precursores; aperfeiçoar o processo de escrituração. O uso exacerbado desses medicamentos é um fato na sociedade atual, gerando preocupação entre as autoridades de saúde, pois, as utilizações prolongadas dos psicofármacos, além de efeitos colaterais indesejáveis, provocam dependência 
química e geram dificuldades quanto ao término do tratamento farmacológico (MOURA et al., 2016; BRASIL, 2007).

O farmacêutico é o profissional indispensável nesse cenário, onde a ocorrência da depressão possui índices cada vez mais elevados e com consequente, aumento do consumo de fármacos antidepressivos, o farmacêutico deve estar atento, principalmente as reações adversas, interações medicamentosa e como profissional da saúde proporcionar a ação multidisciplinar e multiprofissional, tendo a compreensão de que saúde é uma condição integral de bem estar, que assegure cada doente para que este receba a terapêutica necessária para a sua doença, de modo seguro e apropriado, cumprindo às suas responsabilidades perante o tratamento farmacológico (FEGADOLLI et al., 2016). Diante do exposto, este estudo tem por objetivo identificar as razões que motivam à adesão dos pacientes aos medicamentos indicados para tratamento de transtornos depressivos.

\section{METODOLOGIA}

Este estudo trata-se de uma revisão bibliográfica realizada de agosto a novembro de 2018, que tem como propósito responder a seguinte questão: "Quais as razões que motivam ao consumo abuso de antidepressivos?" Segundo Gil (2010) toda pesquisa, para que seja bem elaborada, exige que as ações desenvolvidas sejam efetivamente planejadas sistematicamente e bem organizadas.

Foram pesquisados artigos científicos nas bases de dados: Google Acadêmico, Scientific Eletronic Library Online (Scielo)e no Portal de Periódicos Capes. O número de estudos selecionados foram apresentados na figura 1. Os descritores empregados na pesquisa foram primeiramente consultados junto aos Descritores em Ciências da Saúde (DECS), sendo eles usados isolados ou combinados: "depressão", "antidepressivos" e "assistência farmacêutica”, "depressão and antidepressivos" e "assistência farmacêutica and depressão".

Como critérios de inclusão para a triagem dos estudos foram definidos artigos publicados em português entre janeiro de 2010 a dezembro de 2018, com abordagem envolvendo a depressão (conceito, sintomas e tratamento) e o uso de antidepressivos. Foram excluídos artigos repetidos, resumos, dissertações e teses. 
A triagem dos artigos foi realizada por meio da leitura dos títulos e resumos sendo selecionados 34 artigos (Figura 1). Realizou-se leitura polida do conteúdo bibliográfico obtido, visando agrupá-los por conteúdos de relevância de forma responder os objetivos propostos no presente artigo através das temáticas abordadas em quatro categorias sendo elas: a depressão: epidemiologia, sintomas e diagnóstico; tratamento para o transtorno depressivo; elevado consumo dos antidepressivos; assistência farmacêutica na depressão.

Figura 1: Trajetória da busca nas bases de dados.

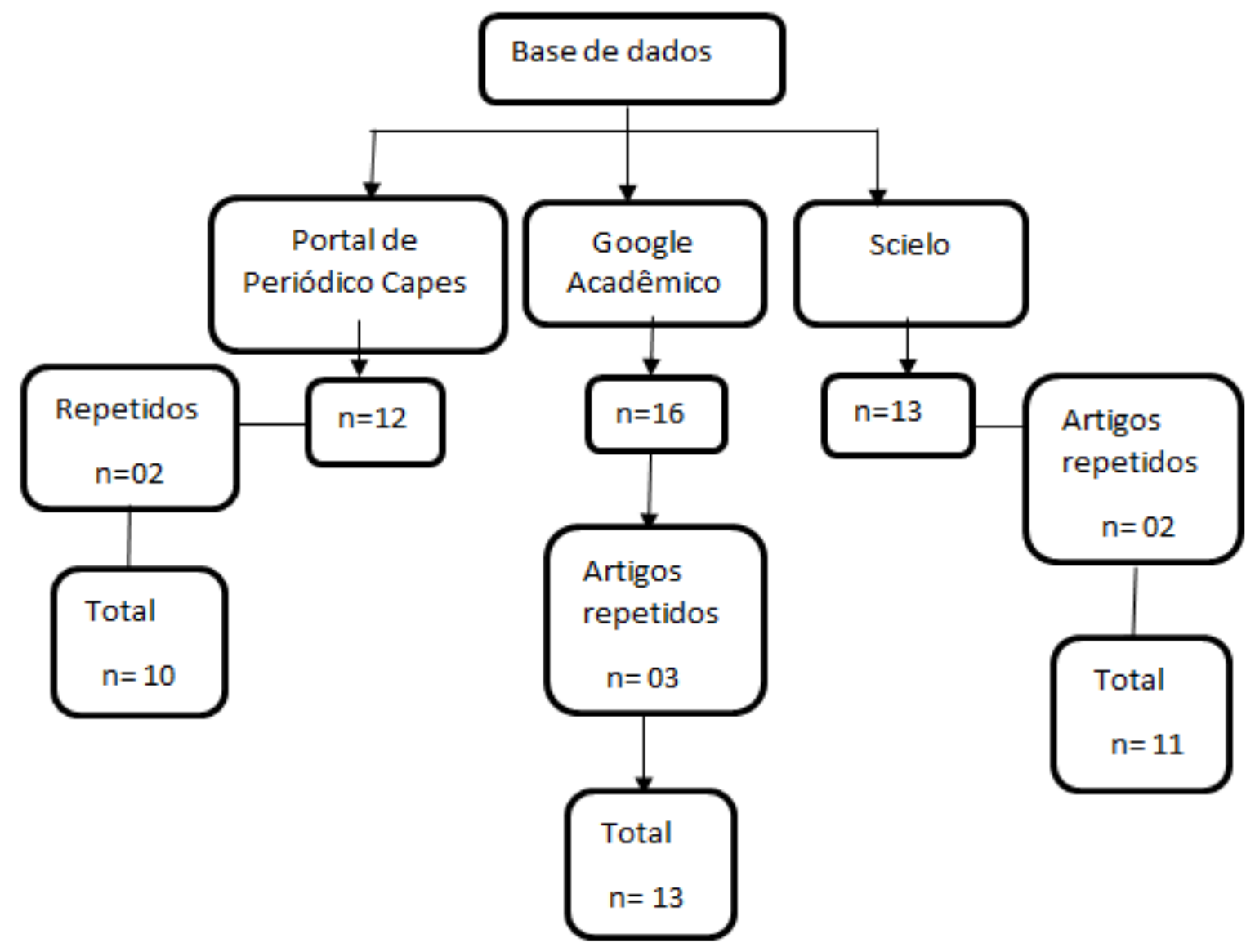

RESULTADOS E DISCUSSÃO

Depressão: sintomas e diagnóstico 
Os dados fornecidos pela Organização Mundial de Saúde (OMS) confirmam que a depressão vem crescendo radicalmente nos tempos atuais, com a probabilidade que em 2020 ela passe a ser seja a segunda doença de maior prevalência no mundo. Estima-se que cerca de $5 \%$ da população mundial (aproximadamente 350 milhões de pessoas) sofra de depressão e que cerca de 10\% a $25 \%$ das pessoas possam apresentar um episódio depressivo em algum momento de sua vida. Para o ano de 2030 estima-se que a depressão se torne a doença mais frequente do mundo, atingindo mais pessoas do que qualquer outro problema de saúde (OMS, 2013; ANTUNES, MONICO, 2015).

O autor Pereira et al (2016) utilizaram os dados estatísticos do Instituto Brasileiro de Geografia e Estatísticas (IBGE) quais corroboram com os apresentados pela OMS, segundo retrata o autor, que no Brasil 7,6\% das pessoas com 18 anos ou mais já foram diagnosticadas, por algum profissional de saúde mental, com depressão. Este dado representa cerca de 11,2 milhões de pessoas, sendo que a prevalência de depressão foi maior no sexo feminino de $(10,9 \%)$ e no sexo masculino (3,9\%). A depressão pode comprometer as atividades diárias das pessoas, entre elas o trabalho, ocasionando o afastamento desses indivíduos das atividades laborais, levando a prejuízos econômicos para os empregadores, e para os cofres públicos, que gastará com medicamentos e atendimentos especializados para essa população. (BRASIL, 2014).

Os autores Andrews et al (2015) e Haberstick (2016) definem a patogenia da depressão à redução nos níveis das monoaminas na fenda sináptica, entre elas a serotonina, que levam à mudança no humor, podendo ocasionar sensações de tristeza. Os estudos realizados atualmente comprovam que a carência de serotonina, afeta o sistema gastrintestinal podendo causar síndrome do intestino irritável, como também favorece o aumento da ansiedade, conduzindo ao distúrbio do sono e na alimentação, causando dores musculares, falta de libido e energia.

Segundo os estudos de Stopa e colaboradores (2015), afirmam que as causas do transtorno do humor evidenciado na depressão são decorrentes de fatores diversos como: genéticos, psicológicos, familiares e sociais. O Manual Diagnóstico e Estatístico de Transtornos Mentais (DSM-V) caracteriza a depressão como um conjunto, sistematizados denominado como "transtornos do humor" e 
"transtornos afetivos" pela Classificação Internacional de Doenças e Problemas Relacionados.

Os sintomas da depressão são descritos pela Fundação Osvaldo Cruz Fiocruz (2015) e a OMS (2015), como uma alteração em quatro campos do funcionamento humano: afetivo, cognitivo, comportamental e fisiológico, sendo então caracterizada por tristeza, perda de interesse em coisas que antes eram prazerosas (anedonia), ausência de prazer, angústia, medo, ideação de culpa, inferioridade, falta de sentido da vida ou desejo de morte, além de distúrbios do sono ou do apetite.

Os estudos de Almeida e Faro (2016) definem de forma ampla os sintomas da depressão diferenciando-a do humor deprimido ocasional. A afirmaram que os sintomas da depressão estão presentes praticamente todos os dias, por um período de mais de duas semanas, devendo o indivíduo apresentar também sintomas adicionais, tais como: apatia, falta de confiança, visões negativas de si e dos outros, alterações no apetite ou peso, sono e atividade psicomotora; fadiga, sentimentos de desvalia ou culpa, energia reduzida; irritabilidade; dificuldade para pensar, concentrar-se ou tomar decisões, perturbação da memória e dificuldade na aprendizagem; isolamento social; pensamentos recorrentes sobre morte ou ideação suicida, planos ou tentativas de suicídio.

Para o diagnóstico da depressão, os estudos realizados por Almeida e Faro (2016) e Almeida (2015) apontam a utilização de um instrumento padronizado para o diagnóstico, onde as escalas de classificação definem o tipo de depressão em distúrbios depressivo leve, moderado ou distúrbio depressivo maior. Os distúrbios depressivos mais comuns são os distúrbios da depressão maior, que apresentam sintomas graves que interferem na capacidade de trabalhar, dormir, concentrar, comer e realizar as atividades diárias.

Almeida (2015) afirmam que, a escala mais utilizadas é o PHQ-9, pois, classifica o tratamento de acordo os sintomas apresentados, permitindo 0 rastreamento contínuo do tratamento e a gravidade dos sintomas, Almeida e Faro (2016) que são as análises dos sintomas que irão classificar os distúrbios depressivos por isso, o diagnóstico se torna complexo. A Associação Americana de Psiquiatria (2014) utiliza o Manual de Diagnóstico e Estatístico das Perturbações Mentais (DSM-V) como ferramenta fundamental para o diagnóstico da depressão. 


\section{Tratamento dos transtornos depressivos}

Os estudos de lbanez et al (2014) apresentam as classes antidepressivas de forma sucinta e objetiva, as classes de antidepressivos mais utilizadas são os inibidores de monoaminaoxidase (IMAO), antidepressivos tricíclicos, os antidepressivos inibidores seletivos da receptação de serotonina e os antidepressivos inibidores seletivos da recaptação de serotonina e norepinefrina.

Conforme Balanescu et al (2017) os antidepressivos inibidores da enzima monoaminaoxidase (IMAO) foram descobertos em 1951, representados pelos fármacos isoniazida e a iproniazida. Eles foram desenvolvidos e usados para o tratamento da tuberculose, observaram então uma alteração do humor, aumentando a euforia dos pacientes tuberculosos, e em 1952 comprovaram que a iproniazida era um inibidor da IMAO. O mecanismo de ação se dá ao inibir a recaptação dos neurotransmissores diminuindo a sua destruição por ação da MAO resultando no aumento do nível dos neurotransmissores na fenda sináptica e consequentemente uma reestabelecendo o humor do doente.

Segundo o estudo de Ferreira e Melo (2018) os antidepressivos tricíclicos (ADT) é uma das classes de antidepressivos mais utilizados. Os estudos de lbanez et al (2014) afirmando que essa descoberta dos ADT ocorreu em 1951, durante a investigação clínica dos isósteros fenotiazínicos. Os autores Ferreira e Melo (2018), Ibanez e colaboradores (2014) afirmam que agem reduzindo a redução da recaptação de serotonina e norepinefrina, aumentando a disponibilidade desses neurotransmissores na fenda sináptica. Há uma predominância da atividade noradrenalina e outros da serotoninérgica, bloqueando os receptores histaminérgicos, colinérgicos e muscarínicos responsáveis pela maioria dos efeitos adversos.

Furukawa et al (2016) em concordância com os autores Prado et al (2017), Stopa et al (2015) afirmam que os Inibidores Seletivos da Recaptação de Serotonina (ISRS) inibem a receptação da serotonina na fenda sináptica, aumentando a liberação da monoamina e, consequentemente, a atividade serotonérgica. Por atuarem de forma seletiva, não exercem ação sobre outras catecolaminas 
(norepinefrina e dopamina). Porém apresentam atividades anticolinérgicas, adrenérgica e histaminérgica.

Os dos autores Furukawa et al (2016), Prado et al (2017), Stopa et al (2015) confirmam que a fluoxetina é o antidepressivo ISRS mais utilizado, sendo que a mesma foi desenvolvida a partir dos Antidepressivos Tricíclicos com o objetivo de diminuir a afinidade pelos receptores adrenérgicos, colinérgicos e histaminérgicos e aumentar a afinidade para as bombas de recaptação da serotonina ou 5hidroxitriptamina (5-HT), fazendo parte dos antidepressivos de terceira geração. A fluoxetina segundo os estudos realizados por Furukawa et al (2016) é um dos antidepressivos mais consumidos atualmente, é comercializado com nome de Prozac®. A estimativa é de aproximadamente 40 milhões de pessoas são usuárias deste medicamento, pelo fato de apresentar baixo custo, boa tolerabilidade e efetividade.

Os estudos de Ferreira e Melo (2018) incluem os Inibidores seletivos da receptação de seretonina e Norepinefrina (ISRN), os fármacos representantes desta classe de antidepressivos são a venlafaxina e a duloxetina. A venlafaxina é um potente inibidor da receptação de serotonina e da norepinefrina, com baixa atividade sobre a dopamina. A duloxetina inibe com especificidade a receptação de serotonina e norepinefrina, com atividade equilibrada entre as duas monoamias. O fármaco é metabolizado pelo fígado, portanto não está indicado para hepatopatas.

Segundo Schenkel et al (2015) deve-se ter cuidado quanto as interações medicamentosas (IM) entre as classes antidepressivas. A fluoxetina quando administrada à amiodarona ou imipramina ou amitriptilina. A I.M fluoxetina e amiodarona pode resultar em um aumento do risco de cardiotoxicidade. A Duloxetina pode apresentar algumas interações medicamentosas entre elas, o ácido salicílico, celecoxibe, clopidogrel, diclofenaco, dipirona, heparina, ibuprofeno, risco de sangramento. Amitriptilina, clorpromazina, imipramina, nortriptilina, causam aumento nos níveis plasmáticos desses medicamentos, podendo levar a efeitos tóxicos. Em pacientes hipertensos deve se ter cautela, o uso deve ser limitado, pois pode ocorrer sangramento gastrointestinal, constipação e distúrbios na ejaculação.

\section{Consumo abusivo de antidepressivos}


Machado e Ferreira (2014) afirmam em seus estudos que os antidepressivos detêm o terceiro lugar entre os fármacos mais vendidos no mundo. $O$ aumento do consumo de antidepressivos, segundo os autores encontram-se relacionando ao surgimento de novas medicações, ampliação das indicações terapêuticas, como o crescimento do diagnóstico das doenças depressivas na população mais jovem. $O$ consumo desses fármacos tende a aumentar com a idade e está associado ao contexto familiar e à saúde mental, prolongamento do tratamento medicamentoso da depressão. Prado et al (2017) afirmam que com envelhecimento da população aumenta-se o índice de depressão, pois a população idosa brasileira nos estudos aponta prevalência de uso de antidepressivo entre 7,2\% a 23,6\% (LOYOLA FILHO et al., 2014; VICENTE et al., 2015).

Segundo o IBGE (2013) no Brasil o maior percentual de casos de depressão ocorre no sexo feminino, o correspondente a 11,2 milhões de mulheres. De acordo com o IBGE (2013), esta prevalência está relacionada às mudanças no papel da mulher na sociedade, tais como o acúmulo de tarefas decorrentes da realização de atividade profissional, em função do aumento da participação no mercado formal de trabalho, além do acúmulo de responsabilidades no cuidado da família e do domicílio. Outros fatores que estão associados ao grande consumo de antidepressivos são: característica sociodemográficas, falta de lazer, baixa escolaridade, situação conjugal, situação econômica e empregatícia.

Uma vez que fazem parte da lista da Relação Nacional de MedicamentosRENAME 2017, e por ter um preço acessível em farmácias particulares os medicamentos antidepressivos são obtidos nas farmácias básicas do S.U.S. Porém, o consumo de antidepressivos não atinge níveis maiores uma vez que segundo Almeida e colaboradores (2015) esses medicamentos são reconhecidos popularmente como medicamentos controlados e possuem seu controle de distribuição e dispensação dos fármacos de controlados, é realizado pelo Sistema de Gerenciamento de produtos controlados (SNGPC) efetivo um controle online sobre produção, circulação, comércio e uso de substâncias ou medicamentos sujeitos a controle especial (BRASIL, 2017).

Existem várias razões que contribuem para o elevado consumo desses fármacos de antidepressivo Rocha e Werlang (2013) associam o elevado consumo ao número de prescrições de medicamentos antidepressivos desnecessários, como 
também da renovação de receita sem reavaliação do paciente, atividades muitas vezes executadas por um médico clínico geral, o que seria função do psiquiatra, porque além de ser o profissional qualificado para tal função, possui mais conhecimento com relação à farmacoterapia. Esses são fatores que contribuem para um tratamento sem sucesso, correlacionados à falta da informação do prescritor ao usuário no que diz respeito ao tratamento farmacológico.

O autor Margarido (2012) ressalta outra razão para o aumento do consumo dos antidepressivos que é a ampliação das indicações, sendo então usados para casos que não tem relação com a depressão, tais como: tensão pré-menstrual, dor física, o mau humor, a ejaculação precoce. Esses sintomas não se relacionam à depressão, mas dão ao indivíduo a ideia de sofrimento e mal-estar onde são atenuados através da terapia medicamentosa antidepressiva.

Sanches e Amarante (2014) relacionam a medicalização a uma atitude consumista, na qual as questões de saúde são consideradas como um mercado, e esse é um fenômeno que tem se intensificado bastante em nossa sociedade, os autores Sanches e Amarante (2014) ressaltam que o uso exagerado e inadequado de medicamentos levou a medicalização, apoiando-se sobre o pensamento, que através da terapia medicamentosa pode se anular o sofrimento que a sociedade pode causar, transformando todo cidadão em consumidor de seus produtos, à indústria farmacêutica alcança pessoas saudáveis que vêm consumindo grande proporção de medicamentos.

Não menos importante que os demais fatores citados, o fator publicitário também contribui para o alto consumo de antidepressivos. Os autores Machado e Ferreira (2014) enfatizam que a publicidade opera no nível do desejo, sob o imperativo do consumo, em que o desejo é alcançado e modificado em mercadoria. O mercado farmacêutico não se finda nos medicamentos (SANCHES, AMARANTE, 2014).

Podem ser exemplos da grandiosidade da atuação do mercado farmacêutico e do incentivo a medicalização médicos psiquiatras norte-americanos, financiados pelos laboratórios farmacêuticos para articular comparações sobre seus medicamentos. Cursos para diagnosticar transtornos mentais são ministrados, revistas médicas são financiadas para a publicação de resultados favoráveis aos medicamentos e empresas de exames médicos crescem desenfreadamente nos 
Estados Unidos com o mercado de diagnósticos de transtornos mentais por meio de imagens cerebrais.

\section{Assistência farmacêutica na depressão}

A Assistência Farmacêutica (AF) segundo Três et al (2013) é uma ferramenta fundamental no tratamento da depressão, permitindo que haja a participação de toda equipe de saúde, possibilitando ao profissional farmacêutico uma dedicação maior ao paciente exercendo a atenção farmacêutica, prestando suporte técnico e orientando com relação ao tratamento farmacoterapêutico, prevenindo dessa forma problemas relacionados a medicamentos (PRM).

Segundo a Agencia Nacional de Vigilância Sanitária a farmacovigilância é definida pela OMS como a ciência e atividades relativas à identificação, avaliação, compreensão e prevenção de efeitos adversos ou qualquer problema relacionado ao uso de medicamentos. Por meio da farmacovigilância o farmacêutico pode realizar à intervenção no que diz respeito ao uso racional de medicamentos, verificando a farmacoterapia, fazendo o acompanhamento do paciente analisando as prescrições, evitando assim que ocorram interações medicamentosas (BRASIL, 2017).

Ferreira e Melo (2018) descrevem o papel do farmacêutico clínico na depressão acrescentando ações importantes como: realizar orientações sobre os medicamentos tanto para o paciente, quanto para os profissionais da saúde, monitorar a farmacoterapia prescrita e intervir, quando for necessário, como reconciliação medicamentosa, via de administração, forma farmacêutica, posologia e administração inadequada, bem como na substituição de medicamentos envolvidos em interações medicamentosas, a fim de garantir a segurança do paciente.

A aquisição dos antidepressivos faz parte do ciclo da assistência farmacêutica onde o farmacêutico é o responsável pelo acesso a estes medicamentos, pois os antidepressivos fazem parte dos Componentes Básicos de Assistência Farmacêutica (CBAF), que são financiados pela União, Estados, Ministério da Saúde, Distrito Federal e Municípios, sendo distribuídos para as farmácias de rede pública em ciclos trimestrais, e são atribuídos para a suplementação da atenção primária para a população da cidade e microrregiões, considerando a necessidade de cada pessoa com relação ao uso de medicamentos por essas razões é tão importante o 
farmacêutico está inserido nos serviços público, sendo este o profissional que entende de medicamentos (OLIVEIRA, FREITAS (2012).

Fegadolli et al (2016) ressaltam que a reorientação das ações de Assistência Farmacêutica, prevista nas políticas públicas de saúde brasileiras, é fundamental para a transformação desse cenário. Um dos pontos fundamentais dessa reorientação é a maior participação do farmacêutico no trabalho em equipe, como especialista em medicamentos, assessorando os demais profissionais quanto à racionalidade e otimização de tratamentos farmacológicos.

Segundo os autores Oliveira e Freitas (2012) a comunicação entre farmacêutico e paciente é primordial no que se refere aos medicamentos utilizados e circunstâncias relacionada ao uso, como, os efeitos colaterais, esses efeitos podem manifestar, levando a um raciocínio de não efetividade por terem efeitos que demoram até semanas para se manifestar. Pode acontecer que o estado de consciência do paciente esteja prejudicado pela doença, sendo necessário que os familiares e cuidadores tenham consciência dos efeitos que a medicação possa desencadear.

A partir dos estudos realizados por Ferreira e Melo (2018) acerca do tratamento da depressão, conclui-se que o farmacêutico possui atribuições de grande importância no trabalho em conjunto com os profissionais da saúde, orientando-os quanto aos riscos que a farmacoterapia pode oferecer, assim como a maneira correta de medicar os pacientes, garantindo a segurança e eficácia do tratamento, colaborando para a redução do número de medicações inadequadas e as devidas interações causadas pelos mesmos.

Almeida et al (2015) salientam que o acesso e o credenciamento dos estabelecimentos farmacêuticos são de responsabilidade do farmacêutico responsável técnico cadastrado com perfil de acesso ao Sistema Nacional de Gerenciamento de Produtos Controlados (SNGPC), na qual todos os medicamentos controlados de uma farmácia e drogaria devem estar escriturados no SNGPC. Para que ocorra um monitoramento adequado e de forma mais ágil, fornecendo informações que contribuem para ações de vigilância sanitária resultando na maior eficiência do uso correto e seguro dos medicamentos conhecidos como controle especial, em especial os antidepressivos. 
Segundo Oliveira e Freitas (2012) a dispensação de antidepressivos devem ser realizada mediante prescrição médica e o farmacêutico é o profissional capacitado para conduzir ações relacionadas aos medicamentos, o seu principal objetivo é garantir a adesão terapêutica, promover o uso racional de medicamentos e oferecer instruções para o paciente, em linguagem clara e objetiva, respeitando o nível de compreensão individual.

\section{CONCLUSÕES}

O trabalho alcançou seu objetivo proposto uma vez que elucidou as razões que motivam o uso elevado de antidepressivos tais como: aumento do envelhecimento da sociedade e as condições sociodemográficas da população; elevados números de prescrições de antidepressivos desnecessários; ampliação das indicações dos antidepressivos para outros agravos que não sejam o transtorno depressivo e a medicalização onde a sociedade procura anular o sofrimento com a terapia medicamentosa.

A depressão é um problema de saúde pública que traz grandes impactos, tanto na vida do paciente ocasionando o afastamento de suas atividades laborais e também economicamente, pois eleva as despesas públicas em relação à aquisição de medicamentos antidepressivos. Para obter um controle eficaz do consumo de antidepressivos o farmacêutico possui um papel fundamental, pois é o profissional responsável pela aquisição dos medicamentos, garantindo o tratamento do transtorno depressivo e assegurando que o medicamento e a dose terapêutica estejam adequados ao paciente. O farmacêutico deve estar inserido nas ações educativas realizadas junto à equipe multidisciplinar, pois estas ações diminuem os índices de consumo abusivo de antidepressivos.

A assistência farmacêutica atua de forma integra no controle da dispensação dos antidepressivos operando junto ao sistema SNGPC e garantindo que o medicamento, a posologia esteja adequada ao paciente, ofertando a este segurança e eficácia no tratamento do transtorno depressivo. O sistema de farmacovigilância permite o monitoramento das reações adversas e as interações medicamentosas, garantindo que os benefícios do tratamento farmacológico sejam maiores que os riscos por eles causados. As informações fornecidas por estes sistemas permitem a 
elaboração de medidas para o controle do consumo de antidepressivos. Os índices de consumo de antidepressivos poderiam estar mais elevados sem o controle do SNGPC e a farmacovigilância.

O farmacêutico deve apresentar um olhar humano aos pacientes depressivos, pois a depressão é o último estágio da dor humana. Deve compreender a subjetividade a pluralidade de cada paciente com atenção, carinho, respeito e dignidade, na qual resultará em um tratamento adequado sem a necessidade de prolongamento do mesmo, diminuindo o abuso das drogas antidepressivas. $O$ SNGPC é uma ferramenta manuseada pelo farmacêutico no controle e fiscalização de medicamentos e substâncias sujeitos a controle especial, onde o medicamento antidepressivo é dispensado com prescrição médica obedecendo à legislação vigente.

\section{REFERÊNCIAS BIBLIOGRÁFICAS}

ANDREWS, P. W. et al. Isserotonin an upper or a downer? The evolution of the serotonergic system and its role in depression and the antidepressant response. Neuroscience and Biobehavioral Reviews, v. 51, p. 164-188, 2015.

ALMEIDA, L.G. R.; FARO, A. Levantamento e principais achados de estudos nacionais sobre a depressão-uma revisão sistemática de literatura. REVIPI, v. 2, n.1, p. 1-16, 2016.

ALMEIDA, F. B. et al. Análises dos dados do sistema nacional de gerenciamento de produtos controlados (SNGPC) na cidade de Cajazeiras-PB. Revista Fama de ciência da saúde, v. 1, n. 1, p. 01-05, 2015.

ANTUNES, S. M; MONICO, L. S. M. Depressão, ansiedade e stress em doentes deprimidos: estudo com a EADS-21. International Journal of Developmental and Educational Psychology INFAD Revista de Psicología, v. 02, n. 01, p. 419-428, 2015.

ASSOCIAÇÃO AMERICANA DE PSIQUIATRIA. Manual Diagnóstico e Estatístico de Transtornos Mentais - DSM-5. 5 ${ }^{\text {a }}$ Edição. Washington: American Psyachiatric Association, 2014.

BRASIL. Secretaria de Ciência, Tecnologia e Insumos Estratégicos, Departamento de

Assistência Farmacêutica e Insumos Estratégicos, Ministério da Saúde. Relação nacional de medicamentos essenciais - RENAME. Ed. Brasília. Ministério da Saúde. 2017. 
BRASIL. Ministério do Planejamento, Orçamento e Gestão. Instituto Brasileiro de Geografia e Estatística. Pesquisa Nacional de Saúde 2013: percepção do estado de saúde, estilos de vida e doenças crônicas, Brasil, Grandes Regiões e Unidades da Federação, v.1. Rio de Janeiro, 2014. Disponível em:<http://biblioteca.ibge.gov.br/visualizacao/livros/liv91110.pdf>. Acesso realizado em: setembro de 2018.

BALANESCU, P. et al. Depression screening in primary care and correlations with comorbidities in Romania. International Medical Journal, v. 23, n. 2, p. 128-131, 2017.

FEGADOLLI, C. et al. Farmacêuticos integrando equipes de cuidado em saúde mental no sistema único de saúde. INTERFACE comunicação saúde educação, v. 20, n. 59, p.1093-1098, 2016.

FERREIRA, K. V.; MELO, N. I. Depressão em idosos: o papel do profissional farmacêutico. Rev. Psicol. Saúde e debate. v.4, nํ01, p.44-60, 2018.

FERREIRA, B. R. S. et al. Projeto sim para a vida: Depressão não é frescura, é doença. Revista UFG. v. 17, n. 20, p. 112-130, 2017.

FIOCRUZ - FUNDAÇÃO OSWALDO CRUZ/Canal saúde, 2015. Disponível em: http://www.canal.fiocruz.br/destaque/index.php?id=722. Acesso em: setembro. 2018.

FURUKAWA, T. A. et al. Eficácia comparativa e aceitabilidade de antidepressivos de primeira geração e segunda geração no tratamento agudo da depressão maior: protocolo para uma metanálise em rede. BMJ Open, v. 6, n. 7, 2016.

GIL, Antonio Carlos. Como Elaborar Projetos de Pesquisa. 5a ed. São Paulo: Atlas, 2010.

HABERSTICK, B. C.et al. Depression, stressful life events, and the impact of variation in the serotonin transporter: Findings from the National Longitudinal Study of Adolescent to Adult Health (Add Health). PloSOne, v. 11, n. 3, p. 1-13, 2016.

IBANEZ, G., et al., Adesão e dificuldades relacionadas ao tratamento medicamentoso em pacientes com depressão. Rev. Bras. Enferm, v. 64, n. 4, p. 556-562, 2014.

LOYOLA, F.I. et al. tendências no uso de antidepressivos entre idosos mais velhos: projeto Bambuí. Revista Saúde Pública. v.48, n. 6, p. 857- 865, 2014.

MACHADO, L. V.; FERREIRA, R.R. A indústria farmacêutica e psicanalise diante da epidemia de depressão; respostas possíveis. Readalyc.org, v. 19, n. 1, p. 135- 144, 2014.

MAGALHÃES, A. E. C. et al. Psicotrópicos: perfil de prescrições de benzodiazepínicos, antidepressivos e anorexígenos a partir de uma revisão sistemática, Electronic Journal of Pharmacy, v. 13, n. 3, p. 111-122, 2016. 
MARGARIDO, F.B. A banalização do uso de ansiolíticos e antidepressivos. Revista de psicologia, v. 15, n. 22, p. 133-146, 2012.

MOURA, D.C.N. et al. Uso abusivo de psicotrópicos pela demanda da estratégia saúde da família: Revisão integrativa da literatura. SANARE, Sobral, v. 15, n. 02, p. 136-144, 2016.

OMS (ORGANIZAÇÃO MUNDIAL DA SAÚDE). The World Health Report - Mental Health: New Understanding, New Hope, 2015.

OLIVEIRA, F. R.A. M.; FREITAS, R. M. Atenção farmacêutica a um portador de depressão. Revista eletrônica de farmácia, v. 9, n. 3, p. 54-66, 2012.

PADILHA, P. D. M. et al. Análise da dispensação de medicamentos psicotrópicos pela rede pública municipal de saúde de Campo mourão/PR. Revista UNINGÁ Review, v. 20, n. 2, p.06-14, 2014.

PRADO, M. A. M. B. et al. Uso de medicamentos psicotrópicos em adultos e idosos residentes em Campinas, São Paulo: um estudo transversal de base populacional. Epidemiol. Serv. Saúde, v. 26, n. 4, p. 747-758, 2017.

ROCHA, B. S.; WERLANG, M.C. Psicofármacos na Estratégia Saúde da Família: perfil de utilização, acesso e estratégias para a promoção do uso racional. Ciência \& saúde coletiva, v. 18, n. 11, p. 3291-3300, 2013.

SKENKEL, M. et al. Interações medicamentosa em usuarios de antidepressivos do sistema público de um município do sul do Brasil. Ciência \& saúde, v. 8, n. 3, p. 107-114, 2015.

SANCHES, V. N. L.; AMARANTE, P. D. C. Estudos sobre o processo de medicalização de crianças no campo da saúde mental. Saúde e debate, v. 38, n. 102, p. 506-514, 2014.

SALOMAO, P. E. A. et al. As Tecnologias de Informação e Comunicação (TIC) no ensino superior. Revista Multidisciplinar do Nordeste Mineiro, v. 1, 2018.

STOPA, S. R. et al. Prevalência do autorrelato de depressão no Brasil: resultado da Pesquisa Nacional de Saúde, 2013. Rev. Bras. Epidemiol, v. 18, n. 2, p. 170-180, 2015.

VICENTE, A. R. T. et al. Anti depressant use and associated factors among the elderly. The Bambuí project. Ciencia saúde colet. v.20, no 12, p. 3797 - 3804, 2015. 